\title{
As latências e amplitude no potêncial evocado miogênico vestibular em crianças
}

\author{
Latencies and amplitude in vestibular evoked myogenic potential in children \\ Latencias y amplitud del potencial miogénico evocado vestibular en niños
}

Recebido: 19/01/2021 | Revisado: 22/01/2021 | Aceito: 25/01/2021 | Publicado: 31/01/2021

\author{
Joab Prudêncio de Sousa Oliveira \\ ORCID: https://orcid.org/0000-0002-4023-0789 \\ Faculdade de Ensino Superior do Piauí, Brasil \\ E-mail: fonojoabprudencio@gmail.com \\ Ysa Karen Santos Macambira \\ ORCID: https://orcid.org/0000-0001-7061-7880 \\ Faculdade de Ensino Superior do Piauí, Brasil \\ E-mail: ysakaren@gmail.com \\ Ruth Raquel Soares de Farias \\ ORCID: https://orcid.org/0000-0002-0988-0900 \\ Faculdade do Ensino Superior do Piauí, Brasil \\ E-mail: ruthraquelsf@gmail.com
}

\begin{abstract}
Resumo
Objetivo: O presente estudo teve como objetivo observar se existem diferenças significativas para as latências do VEMP entre crianças e adultos. Metodologia: O estudo trata-se de uma revisão sistemática, com abordagem de natureza quantitativa, os descritores utilizados na pesquisa foram: (Child OR Childhood OR Children OR Pediatric) AND (vestibular evoked myogenic potential OR VEMP OR cVEMP OR oVEMP). A seleção dos estudos e extração dos dados foi realizada apenas por dois pesquisadores, onde os valores médios de latência e amplitude do teste VEMP foram os desfechos procurados nos artigos. Resultados: Nos estudos selecionados, um total de 135 indivíduos foram estudados para a avaliação das latências n1 e p1 do oVEMP, dos quais 62 eram crianças e 73 eram adultos, já para avaliação das latências p13 e n23 de cVEMP foram estudados 50 pessoas, sendo 25 crianças e 25 adultos. Nos procedimentos foram utilizados intensidade entre 100 a $105 \mathrm{dBnHL}$, com frequência de $500 \mathrm{~Hz}$ em todos os estudos. As latências dos componentes n1 e p1 e amplitude do oVEMP não possuem diferenças significativas entre as idades, porém nos componentes do cVEMP, é possível verificar uma diferença significativa no componente p13. Conclusão: Os valores das latências do componente de onda e amplitude de oVEMP em crianças não apresenta diferenças significativas em adultos enquanto no componente p13 do cVEMP possuem diferenças significativas por fator de idade.
\end{abstract}

Palavras-chave: Potencial evocado miogênico vestibular; Crianças; Potencial evocado miogênico vestibular ocular; Potencial evocado miogênico vestibular cervical.

\begin{abstract}
Objective: The present study has the objective of observing that there are significant differences for the latencies of VEMP between children and adults. Methodology: The study is about a systematic review, with a quantitative nature approach, the descriptors used in the research form: (Child OR Childhood OR Children OR Pediatric) AND (vestibular evoked myogenic potential OR VEMP OR cVEMP OR or VEMP). A selection of two studies and extraction of two data was carried out by only two researchers, where the mean values of latency and amplitude of the VEMP test were obtained from the results obtained from us. Results: We selected studies, a total of 135 individuals for latency studied for a valuation of latencies $\mathrm{n} 1$ and $\mathrm{p} 1$ of oVEMP, two quais 62 eram children and 73 eram adults, já for valuation of latencies p13 and n 23 of cVEMP foram studied 50 people, sendo 25 children and 25 adults. Our procedures were used intensities between 100 to $105 \mathrm{dBnHL}$, with a frequency of $500 \mathrm{~Hz}$ in all studies. As the latencies of two components $\mathrm{n} 1$ and $\mathrm{p} 1$ and amplitude of oVEMP do not have significant differences between entities, especially cVEMP components, it is possible to verify a significant difference not component p13. Conclusion: The values of the latencies of the wave and amplitude component of oVEMP in children do not show significant differences in adults when there is no p13 component of cVEMP that have significant differences by factor of life.
\end{abstract}

Keywords: Vestibular evoked myogenic potential; Children; Ocular vestibular evoked myogenic potential; Cervical vestibular evoked myogenic potential.

\section{Resumen}

Objetivo: O presente estudo teve como objetivo observar se existem diferenças significativas para as latências do VEMP entre crianças e adultos. Metodología: $\mathrm{O}$ estudo trata-se de uma revisão sistemática, com abordagem de natureza quantitativa, os descritores utilizados na pesquisa foram: (Child OR Childhood OR Children OR Pediatric) AND (vestibular evocado myogenic potencial OR VEMP OR cVEMP OR oVEMP). A seleção dos estudos e extração dos dados fori realiza apenas por dois pesquisadores, onde os valores médios de latência y amplitude do teste VEMP 
forram os desfechos procurados nos artigos. Resultados: Nos estudos selecionados, um total de 135 indivíduos foram estudados para a avaliação das latências n1 e p1 do oVEMP, dos quais 62 eram crianças e 73 eram adultos, já para avaliação das latências p13 e n23 de cVEMP foram estudados 50 pessoas, sendo 25 crianças e 25 adultos. Nos procedimentos foram used intensidade entre 100 a $105 \mathrm{dBnHL}$, com frequência de $500 \mathrm{~Hz}$ em todos os estudos. Como latências dos componentes n1 e p1 e amplitud hacen oVEMP não possuem diferenças significativas entre as idades, porém nos componentes do cVEMP, é posível verificar uma diferença significativa no componente p13. Conclusión: Los valores de las latências del componente de onda y la amplitud de oVEMP en crianças não apresenta diferenças significativas em adultos enquanto no componente p13 do cVEMP possuem diferenças significativo por fator de idade.

Palabras clave: Potencial miogénico evocado vestibular; Niños; Potencial miogénico evocado vestibular ocular; Potencial miogénico evocado vestibular cervical.

\section{Introdução}

Os primeiros estudos a cerca do Potencial Evocado Miogênico Vestibular (VEMP), foram feitos em 1964 e em 1969 , através de respostas miogênicas médias evocadas por estímulos pós-auriculares mediante a estimulação de tone burst, onde concluiram que os efeitos dos estímulos eram provenientes do sáculo vestibular (Backford, et al., 1964). Hoje sabemos que o potencial evocado miogênico vestibular é um teste de avaliação da função dos órgãos otolíticos, podendo fornecer-nos informações sobre a função do sáculo, da divisão inferior do nervo vestibular, do utrículo e da divisão superior do nervo vestibular, (Cal et al., 2016) possuindo características bastante favoráveis ao utilizar, como por exemplo o baixo custo, ausência de efeitos colaterais significativos, a rapidez e ausência desconforto ao paciente (Colebatch, 2001).

Tanto o Potencial Evocado Miogênico Ocular (oVEMP), quanto Cervical (cVEMP) podem ser realizados através de qualquer aparelho capaz de registrar evocados de média latência (Oliveira, et al., 2008), medindo o tempo de estímuloresposta, classificando como normal ou alterado de acordo com o tempo de duração e morfologia da onda elétrica gerada (Welgampola, 2008; Colebath, 2001).

As ondas eletromiográficas do registro de potencial se definem pelas seguintes características: Latência (o tempo que trancorre desde a estimulação até a resposta, seja em valor negativo ou positivo), morfologia da onda e amplitude pico a pico (diferencia os valores entre o ponto mais positivo e mais negativo da onda), sendo a latência o mais utilizado na análise das respostas (Guillén, et al., 2005, Rosegreen, et al., 2010).

O traçado obtido no exame com estimulos sonoros é constinuido de dois complexos de ondas bifásicas. O primeiro potencial apresenta pico positivo com latência média de 13 milisegundos (ms), seguido de um pico negativo com latência média de 23 ms, denominado como P13-N23 ou P14-N21 ou P1-N2 (Halmagyi, Colebath, 1994; Ochi, et. al., 2001).

Latências do cVEMP existem para recém-nacidos no dia $5^{\circ}$ dia após seu nascimento, indicando que o sistema saculocólico se desenvolve no início da vida, porém com latências abaixo do que é encontrado em adultos (Young, et al., 2007). Já no oVEMP só é possível obter informações a partir dos 12 meses de idade, tendo mais confiabilidade a partir dos 3 anos (Wang, 2012), porém sem diferenças significantes dos valores de latência em jovens adultos (Hsu, 2009). Pouco se conhece a respeito das diferenças entre as latências do VEMP entre adultos e crianças, sendo assim, o objetivo deste estudo foi observar de sistematicamente se existem diferenças entre as latências do VEMP de crianças e adultos.

\section{Metodologia}

O estudo trata-se de uma revisão sistemática, com abordagem de natureza com abordagem de natureza qualiquantitativa, pois “os resultados numéricos são complementados por resultados qualitativos” (Pereira, et. al., 2018, p.100)., onde teve como base o seguinte questionamento: Existem diferenças para as latências do cVEMP e do oVEMP entre crianças e adultos? Essa questão da pesquisa foi elaborada com base na estratégia PICO (PatientorProblem, Intervention, ControlorComparasion, Outcomes). 
Os descritores utilizados na pesquisa, foram: (Child OR Childhood OR Children OR Pediatric) AND (vestibular evoked myogenic potential OR VEMP OR cVEMP OR oVEMP). Estratégia de busca encontra-se na (Tabela 1).

As buscas foram realizadas entre os meses de setembro, outubro de 2020, nas seguintes bases de dados: PubMed, ScienceDirect, BVS (lilacs), SciELO, Scopus, Circumpolar Health Bibliographic Database, Embase, Web of Science e Cochrane Central Register of Controlled Trials (CENTRAL). Além das bases de literatura cinzenta: OpenGrey, DissOnline, The New York Academy of Medicine, ClinicalEvidence.com e ClinicalTrials.gov. Não houve delimitação de idioma ou data de publicação.

Tabela 1. Estratégia de busca usada para todas as bases de dados.

\#1 (Child OR Childhood OR Children OR Pediatric) AND (vestibular evoked myogenic potential OR VEMP OR cVEMP OR oVEMP).

Fonte: Autores (2020)

Os critérios de inclusão foram: estudos observacionais do teste potencial evocado miogênico vestibular, em grupos de crianças com audição e sistema vestibular integros, grupos controle, com idade entre 0 a 14 anos, com ao menos resultados de latência e/ou amplitude e comparativo com adultos. Os critérios de exclusão foram: estudos com patologias associadas, grupos com idade superior a 14 anos e artigos sem comparativos com adultos. Também foram excluídos artigos repetidos nas bases de dados, artigos de revisão de literatura.

Após a consulta às bases de dados e aplicação das estratégias de busca foi realizada uma análise minuciosa de todas as publicações encontradas considerando os critérios de inclusão para determinar sua elegibilidade e inclusão no estudo. Feita a análise, 7 artigos que atenderam aos critérios de inclusão foram selecionados para revisão sistemática.

A seleção dos estudos e extração dos dados foi realizada apenas por dois pesquisadores, onde os valores médios de latência e amplitude do teste VEMP foram os desfechos procurados nos artigos. Os textos completos foram lidos e avaliados na íntegra.

Além dos dados do desfecho, foram extraídos os seguintes dados de cada estudo: primeiro autor, ano de publicação, local, as faixas etárias de cada grupo, o número de sujeitos em cada grupo, frequência e o tipo de estimulo usado. 


\section{Resultados}

Figura 1. fluxograma de busca e seleção dos artigos.

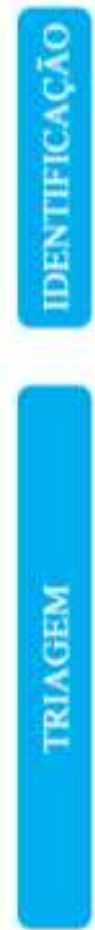

Número de artigos identificados nas bases de dados
\[ (\mathrm{n}=1985) \]
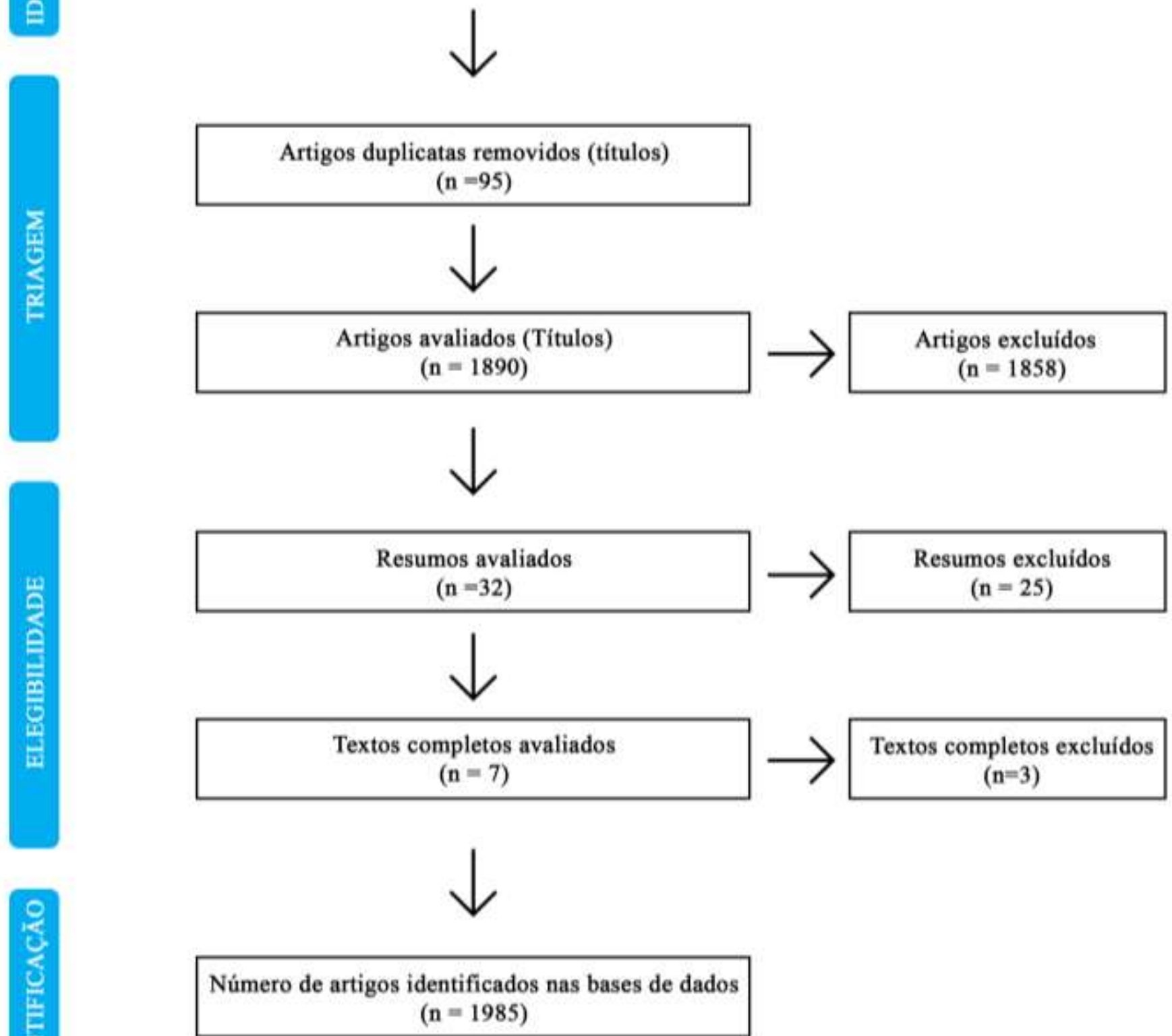

Fonte: Prisma (2009).

O fluxograma da seleção dos artigos é mostrado na figura 1. Incluindo os descritores em todas as bases de dados, foi obtido um total de 1.985 artigos científicos, 95 foram excluídos por duplicatas, e 1.858 por ser irrelevante para a pesquisa. Feita a triagem de títulos, 32 resumos foram lidos e destes 7 foram selecionados para leitura do texto completo. Após a leitura 
de texto completo, 3 artigos foram excluídos de acordo com os critérios de elegibilidade relacionados a faixa etária(Tabela 2). Com isso, 4 artigos foram considerados elegíveis para a pesquisa (Tabela 3).

Tabela 2. Textos completos excluídos da pesquisa.

\begin{tabular}{cccc}
\hline Autores & Local & Ano & Motivo \\
\hline Piccioti, et al. & Itália & 2013 & Faixa etária \\
Beshr et al. & Égito & 2019 & Faixa etária \\
Mendes et al. & Croácia & 2017 & $\begin{array}{c}\text { Sem grupo } \\
\text { controle }\end{array}$ \\
\hline
\end{tabular}

Fonte: Autores (2021)

Tabela 3. Características dos estudos incluídos.

\begin{tabular}{|c|c|c|c|c|c|c|c|}
\hline Autores & Tipo & $\begin{array}{c}\text { Grupo } \\
\text { Crianças }\end{array}$ & Idade & $\begin{array}{c}\text { Grupo } \\
\text { controle }\end{array}$ & Idade & Frequência & Estímulo \\
\hline $\begin{array}{l}\text { Y. -S. Hsu } \\
\text { et. al. } \\
\text { (2009) }\end{array}$ & oVEMP & 15 & $\begin{array}{c}3 \text { a } 13 \\
\text { anos }\end{array}$ & 15 & $\begin{array}{c}24 \text { a } 33 \\
\text { anos }\end{array}$ & 105dBNPS & TB $500 \mathrm{~Hz}$ \\
\hline $\begin{array}{l}\text { Y. -S. Hsu } \\
\text { et. al. } \\
(2009)\end{array}$ & cVEMP & 15 & $\begin{array}{c}3 \text { a } 13 \\
\text { anos }\end{array}$ & 15 & $\begin{array}{c}24 \text { a } 33 \\
\text { anos }\end{array}$ & $105 \mathrm{dBNPS}$ & TB $500 \mathrm{~Hz}$ \\
\hline $\begin{array}{l}\text { Ch. Chou } \\
\text { et. al. } \\
(2012)\end{array}$ & oVEMP & 15 & $\begin{array}{c}3 \text { a } 14 \\
\text { anos }\end{array}$ & 18 & $\begin{array}{c}24 \text { a } 28 \\
\text { anos }\end{array}$ & - & TB $500 \mathrm{~Hz}$ \\
\hline $\begin{array}{l}\text { Rodriguez } \\
\text { et. al. } \\
\text { (2018) }\end{array}$ & oVEMP & 10 & $\begin{array}{l}4 \text { a } 9 \\
\text { anos }\end{array}$ & 10 & $\begin{array}{c}20 \text { a } 29 \\
\text { anos }\end{array}$ & 100dBNPS & TB $500 \mathrm{~Hz}$ \\
\hline $\begin{array}{l}\text { Rodriguez } \\
\text { et. al. } \\
(2018)\end{array}$ & cVEMP & 10 & $\begin{array}{l}4 \text { a } 9 \\
\text { anos }\end{array}$ & 10 & $\begin{array}{c}20 \text { a } 29 \\
\text { anos }\end{array}$ & 100dBNPS & TB $500 \mathrm{~Hz}$ \\
\hline $\begin{array}{l}\text { Kuhn } \\
\text { et. al. } \\
\text { (2019) }\end{array}$ & oVEMP & 22 & $\begin{array}{l}3 \text { a } 9 \\
\text { anos }\end{array}$ & 30 & $\begin{array}{c}24 \text { a } 40 \\
\text { anos }\end{array}$ & 105dBNPS & TB $500 \mathrm{~Hz}$ \\
\hline
\end{tabular}

Fonte: Autores (2021)

Entre os estudos selecionados, os quatro avaliaram as latências do oVEMP, enquanto dois destes avaliavam juntamente o cVEMP. Os quatro artigos apresentavam dados de média e desvio padrão para os componentes de latência do VEMP, para o grupo controle e para o grupo de crianças (Quadro 01). Destes, todos os autores afirmavam não haver diferença significativa entre os grupos nas latencias p1 e n1, porém dois confirmavam diferenças de latência p13 nos grupos em decorrência da faixa etária. Todos os dados estão disponíveis na Tabela 03. 
Quadro 1. Médias e desvios-padrão das latências e amplitudes n1/n23 e p1/p13, para cada grupo e estudo.

\begin{tabular}{|c|c|c|c|c|c|c|c|}
\cline { 2 - 7 } \multicolumn{2}{c|}{} & \multicolumn{2}{c|}{$\begin{array}{c}\text { Média das latências n1/n23 } \\
\text { (DP) }\end{array}$} & \multicolumn{2}{c|}{$\begin{array}{c}\text { Média das latências p1/p13 } \\
\text { (DP) }\end{array}$} & \multicolumn{2}{c|}{$\begin{array}{c}\text { Média das amplitudes } \\
\text { (DP) }\end{array}$} \\
\hline Autores & Tipo & $\begin{array}{c}\text { Grupo } \\
\text { crianças }\end{array}$ & $\begin{array}{c}\text { Grupo } \\
\text { controle }\end{array}$ & $\begin{array}{c}\text { Grupo } \\
\text { crianças }\end{array}$ & $\begin{array}{c}\text { Grupo } \\
\text { controle }\end{array}$ & $\begin{array}{c}\text { Grupo } \\
\text { crianças }\end{array}$ & $\begin{array}{c}\text { Grupo } \\
\text { controle }\end{array}$ \\
\hline $\begin{array}{c}\text { Y. -S. Hsu } \\
\text { et. al. } \\
(2009)\end{array}$ & oVEMP & $11,1 \pm 0,9$ & $11,2 \pm 0,9$ & $16,1 \pm 1,2$ & $16,3 \pm 1,0$ & $7,0 \pm 2,9$ & $9,1 \pm 5,9$ \\
\hline $\begin{array}{c}\text { Y. -S. Hsu } \\
\text { et. al. } \\
(2009)\end{array}$ & cVEMP & $21,1 \pm 2,1$ & $21,9 \pm 1,6$ & $13,8 \pm 1,3$ & $1,6 \pm 1,4$ & $122 \pm-$ & $105 \pm-$ \\
\hline $\begin{array}{c}\text { Ch. Chou } \\
\text { et. al. } \\
(2012)\end{array}$ & oVEMP & $8,0 \pm 0,7$ & $8,4 \pm 0,7$ & $12,2 \pm 1,5$ & $12,7 \pm 1,2$ & $16,1 \pm 19,81$ & $15,2 \pm 8,3$ \\
\hline $\begin{array}{c}\text { Rodriguez } \\
\text { et.al. } \\
(2018)\end{array}$ & oVEMP & $19,25 \pm 2,36$ & $22,20 \pm 1,77$ & $12,25 \pm 1,46$ & $13,92 \pm 1,08$ & $360,60 \pm 185,7$ & $223,7 \pm 99,18$ \\
\hline $\begin{array}{c}\text { Rodriguez } \\
\text { et. al. } \\
(2018)\end{array}$ & cVEMP & $10,59 \pm 1,64$ & $10,10 \pm 1,03$ & $15,31 \pm 2,40$ & $14,56 \pm 1,18$ & $12,06 \pm 8,36$ & $15,59 \pm 1,64$ \\
\hline $\begin{array}{c}\text { Kuhn } \\
\text { et.al. } \\
(2019)\end{array}$ & oVEMP & $10,9 \pm 1,1$ & $10,98 \pm 0,41$ & $15,0 \pm 1,3$ & $15,2 \pm 0,97$ & $15,3 \pm 13,4$ & $13,9 \pm 6,7$ \\
\hline
\end{tabular}

Fonte: Autores (2021).

No Quadro 1, pode ser visualizado os dados das médias e desvio padrão de latência e amplitude n1-p1 encontrados em cada estudo. Pode ser observado que um estudo não apresenta valores de desvio padrão, investigando apenas amplitude pico a pico. Ao avaliar os estudos, é observado que as médias de latência n1 e p1 em crianças normais e controle(adultos) saudáveis não mostraram diferenças entre si quando utilizado o oVEMP, enquanto nos valores da latência p13 do cVEMP, é possível observar uma alteração significativa, definindo assim que há diferenças nas latências do potencial evocado miogenico vestibular entre crianças e adultos.

\section{Discussão}

Devido ao recente aumento de estudos na área de potenciais evocados miogênicos vestibulares, esta revisão destaca estudos publicados entre 2009 e 2019. Dentro disso, o presente estudo buscou mostrar resultados notáveis e relevantes que comparem os valores de latência e amplitude entre crianças e adultos afim de obter um comparativo e possível padrozinação de valores entre indivíduos abaixo de 14 anos. Os principais resultados desta revisão sistemática fundamentaram-se em 4 artigos, nos quais envolveram dados coletados de estudos primários acerca do potencial evocado miogênico vestibular ocular e cervical.

Em relação aos protocolos de teste, os artigos selecionados utilizaram estimulos de forte intensidade, variando de 100 a 105 dBnHL, tendo apenas um estudo não especificando a intensidade utilizada (Chou, et. al., 2012). A maioria optou evocar o VEMP com estímulos de Toneburst, apresentando $500 \mathrm{~Hz}$ de frequência, por ser mais utilizada clinicamente e com resposta mais homogênea e constante. (Wu Ch, et. al., 1999)

De acordo com os resultados, as latências dos componentes n1 e p1 e amplitude do oVEMP não possuem diferenças significativas entre as idades (Hsu, et. al., 2009; Chou, et al., 2012; Rodriguez, et. al. 2018; Kuhn, et. al., 2019), porém nos 
componentes do cVEMP, é possível verificar uma diferença significativa no componente p13(Hsu. et.al., 2009; Rodriguez, et. al., 2018), relacionado ao fato do comprimento do pescoço das crianças serem menores, aumentando a velocidade de condução pela diminuição do comprimento do trajeto do reflexo saculo-cólico, levando a latências de p13 muito precoces(Kuhn, et. al., 2019). Apesar do estudo ser voltado para crianças de 0-14 anos, o principal motivo dos autores utilizarem apenas indivíduos acima de 3 anos é a metodologia, no oVEMP para uma efetividade maior é necessário que o olhar seja fixo para cima, coisa que crianças abaixo de 3 anos possuem dificuldade ao realizar, assim causando dificuldade na fidelidade do exame, enquanto no cVEMP, as latências não são fixas por conta de fatores estruturais relacionados a idade corpórea, fora o desenvolvimento vestibulo-cólico que se desenvolve até os 3 anos de idade, sendo assim a idade essencial para se obter um exame fidedigno(Chou, et. al., 2012).

\section{Considerações Finais}

Os achados demonstram que os valores das latências do componente de onda e amplitude de oVEMP em crianças, não apresentaram alterações quando comparado com adultos, enquanto no componente p13 do cVEMP possuem diferenças significativas por fator de idade. Porém, a quantidade de artigos que envolve crianças e grupo controle com valores de latência e amplitude do potencial evocado miogenico vestibular encontrados para essa pesquisa é relativamente pequeno.

Sabendo disso, constata-se que é necessário o desenvolvimento de novas pesquisas que abordem a temática em questão e/ou que apresentem um potencial similar com o que foi debatido, viabilizando assim uma construção científica mais apurada embasada em um maior percentual de estudos sobre o tema, uma vez que a escassez de estudos científicos sobre uma determinada linha de pesquisa dificulta a construção da mesma. Partindo dessa premissa o desenvolvimento de estudos sobre a temática, com a faixa etária e grupos populacionais abordados faz-se de incomensurável relevância para que se compreenda integral e fidedignamente o uso do potencial evocado miogênico vestibular em crianças e sua confiabilidade.

\section{Referências}

Bickford, R. G., Jacobson, J. L., Cody, D., \& Thane, R. (1964) Nature of Averaged Evoked Potentials To Sound and Other Stimuli in Man. Ann NY Acad Sci, [S. l.], p. 204-223.

Chou, C. H., et. al. (2012). Ocular vestibular-evoked myogenic potentials via bone-conducted vibration in children. Clinical Neurophysiology 123 , $1880-1885$.

Cody, D., Thane, R., \& Bickford, R. G. (1968). Averaged Evoked Myogenic Responses in Normal Man. The Laryngoscope, $400-416$.

Guillén, V., et. al. (2005). Potencial vestibular miogénico evocado: un aporte al conocimiento de la fisiología y patología vestibular. Patrones cuantitavos en la población normal. Acta Otorrinolaringol Esp., [S. l.], 349-353, 2005.

Halmagyi, G. M., Colebatch, J. G., Curthoys, I. S. (1994). New tests of vestibular function. Baillière's Clinical Neurology, 485-500.

Kuhn, et. al. (2018). Ocular Vestibular Evoked Myogenic Potentials: Normative Findings in Children Journal of the American Academy of Audiology. Volume 29.

Menezes, P. L. et. al. (2018) Tratado de Eletrofisiologia da Audição. Booktoy.

Ochi, K., Ohashi, T., \& Nishino, H. (2001). Variance of vestibular-evoked myogenic potentials. Laryngoscope, $522-527$.

Rodriguez, et. al. (2018). Air-Conducted Vestibular Evoked Myogenic Potential Testing in Children, Adolescents, and Young Adults: Thresholds, Frequency Tuning, and Effects of Sound Exposure. Ear \& Hearing.

Wang, S., Hsieh, W., \& Young, Y. (2012) Development of Ocular Vestibular-Evoked Myogenic Potentials in Small Children. The Laryngoscope, [S. l.], 512517.

Welgampola, M. S. (2008) Evoked potential testing in neuro-otology. Curr Opin Neurol., 29-35.

Y.-S. H., et al. (2009). Ocular vestibular-evoked myogenic potentials in children using air conducted sound stimulation. Clinical Neurophysiology 120, 13811385 .

Young, Y., Chen, C., Hsieh, W., \& Wang, S. (2007). Development of vestibular evoked myogenic potentials in early life. European Journal Of Pediatric Neurology, [S. l. ], 235-239. 